\title{
Cluster Preface: Modern Nickel-Catalyzed Reactions
}

\author{
Ruben Martin*a,b \\ Gary A. Molander*c \\ anstitute of Chemical Research of Catalonia (ICIO), The Barcelona \\ Institute of Science and Technology, Av. Països Catalans 16, 43007 \\ Tarragona, Spain \\ rmartinromo@iciq.es \\ ICREA, Passeig Lluís Companys 23, 08010 Barcelona, Spain \\ 'Department of Chemistry, University of Pennsylvania, Philadelphia, \\ Pennsylvania 19104-6323, United States \\ gmolandr@sas.upenn.edu \\ Published as part of the Cluster \\ Modern Nickel-Catalyzed Reactions
}

Received: 17.08.2021

Accepted after revision: 17.08.2021

Published online: 02.09 .2021

DOI: 10.1055/s-0040-1720393; Art ID: st-2021-u6666-c

Ruben Martin is a professor at the Institute of Chemical Research of Catalonia (ICIQ), Tarragona, Spain. He received his Ph.D. in 2003 from the University of Barcelona under the guidance of Prof. Antoni Riera. In 2004, he moved to the Max-Planck Institut für Kohlenforschung as a Humboldt postdoctoral fellow with Prof. Alois Fürstner. In 2005, he undertook further postdoctoral studies at MIT with Prof. Stephen L. Buchwald as a MEC-Fulbright fellow. In 2008, he began his independent career as an assistant professor at the ICIQ (Tarragona). In 2013, he was promoted to associate professor and shortly after to ICREA Research Professor. Ruben Martin has focused his career on designing synthetically useful Ni-catalyzed methodologies for streamlining the preparation of added-value chemicals from simple precursors without losing sight of mechanistic considerations, when appropriate.

Gary A. Molander is a professor at the University of Pennsylvania, Philadelphia, United States. He completed his undergraduate studies in chemistry at lowa State University under the tutelage of Prof. Richard C. Larock. He earned his Ph.D. at Purdue University under the direction of Prof. Herbert Brown and undertook postdoctoral training with Prof. Barry Trost at the University of Wisconsin, Madison. He began his academic career at the University of Colorado, Boulder, moving to the University of Pennsylvania in 1999, where he is currently Professor of Chemistry. His research interests have focused on the utilization of organolanthanides, Pd-catalyzed cross-coupling reactions with trifluoroborate salts, and the merger of photoredox catalysis and Ni catalysis for tackling a priori uphill transformations under visible-light irradiation for accessing valuable scaffolds in both academic and pharmaceutical laboratories.

Nickel has historically been surrounded by a cloud of mystery. While trying to extract copper from a mineral deposit, miners found in the eighteenth century that it was not only impossible to do, but also they became seriously ill. Convinced that the deposit was the mischievous work of the devil, miners named it 'Kupfernickel' or 'Old Nick's Copper', a colloquial name for the devil according to Saxon mythology. Years later, Axel Fredrik Cronstedt managed to identify arsenic as being responsible for the miners' illnesses, together with a new metal that he called nickel. It was not until the twentieth century that the scientific community recognized the potential of nickel as a component in catalysts for synthetic organic endeavors. In 1922, Nobel

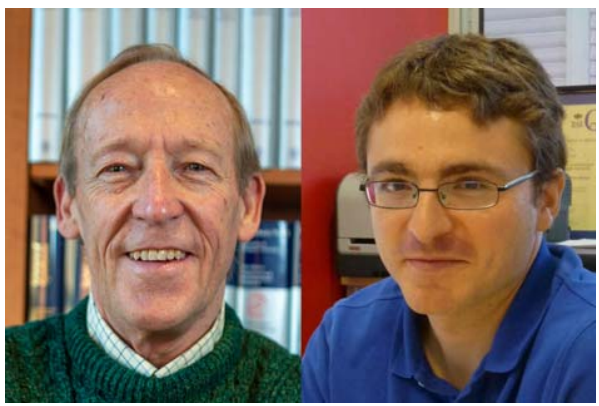

Laureate Paul Sabatier had already noticed the outstanding catalytic activity of nickel together with its Achilles' heel, which was nothing but the difficulty to control its promiscuous reactivity, suggesting that $\mathrm{Ni}$ should not be suited for synthetic methods.

However, such an observation was probably premature, as the genesis of cross-coupling reactions can be traced back to the seminal work of Kumada and Corriu in 1972, ${ }^{1}$ who independently described the ability of nickel catalysts to enable the coupling of Grignard reagents with alkenyl and aryl halides. Although Ni-catalyzed reactions were overshadowed by the impressive applications of Pd-based technologies discovered shortly thereafter, recent years have witnessed tremendous activity in the area of nickel catalysis. The reasons for such a renaissance cannot be merely attributed to Ni being a cheaper substitute of Pd. Indeed, $\mathrm{Ni}$ is more electropositive than Pd; therefore, oxidative addition reactions occur more rapidly at Ni centers, an observation that has turned into a strategic advantage for activating particularly strong $\sigma$-bonds that cannot be accessed with Pd catalysts. The binding of alkenes to Ni complexes is exceptionally strong when compared to Pd, and it comes as no surprise that the latter have become privileged catalysts for the mono- and difunctionalization of olefins. Unlike classical polar $\mathrm{Pd}(0) / \mathrm{Pd}(\mathrm{II})$ mechanisms, multiple catalytic regimes are viable with Ni catalysis. This observation is related to their propensity to trigger single-electron transfer processes, an aspect of utmost synthetic relevance in the context of photoredox catalysis. In addition, the low bond dissociation energy of the $\mathrm{C}-\mathrm{Ni}$ bond provides the fundamental basis for tackling uphill transformations that would otherwise be beyond reach.

In this SYNLETT cluster, we have contributions from authoritative experts in the area of $\mathrm{Ni}$ catalysis, ranging from technologies occurring via two-electron manifolds to photoredox endeavors that operate by the intermediacy of open-shell species. Specifically, Murakami illustrates the ability of a Ni catalyst to streamline the preparation of 1,3dienes from simple carbonyl groups. ${ }^{2}$ Stradiotto shows the potential of DalPhos ligands for effecting a general N-arylation of amides with (hetero)aryl electrophiles, ${ }^{3}$ while Liu, ${ }^{4}$ 
Yin $^{5}$ and $\mathrm{Shi}^{6}$ offer innovative protocols for the rapid preparation of biaryls via $\mathrm{C}-\mathrm{O}$ bond functionalization, Ullman cross-coupling reactions with two $\mathrm{sp}^{2}$ hybridized organic halides, and Hiyama-type technologies for accessing vinylated arenes, respectively. Yorimitsu describes the development of a general Negishi-type cross-coupling reaction of easily accessible trialkylsulfonium salts, ${ }^{7}$ while Fleischer and Berkefeld report the utilization of a Ni catalyst to cleave simple allyl ethers chemoselectivity via formal $\mathrm{sp}^{3} \mathrm{C}-\mathrm{O}$ functionalization. ${ }^{8}$ The groups of Mei and Fang ${ }^{9}$ and Wang ${ }^{10}$ demonstrate that Favorskii rearrangements and reductive allylic defluorinative coupling reactions are not only within reach, but also allow rapid access to cyclopropanes decorated with nitriles or aliphatic alcohols possessing a difluoroalkene isostere on the alkyl side chain. Ogoshi ${ }^{11}$ and Kimura, ${ }^{12}$ respectively, describe the development of carbonylation and carboxylation methods for rapidly and reliably accessing $\gamma$-lactams and carboxylic acids possessing alkenes on the side chain.

Meanwhile, Nishihara ${ }^{13}$ and Yamaguchi ${ }^{14}$ demonstrate remarkable decarbonylation events for incorporating alkyne motifs and nitriles into arenes via $\mathrm{C}-\mathrm{F}$ and $\mathrm{C}-\mathrm{O}$ bond cleavage. Zhang, ${ }^{15}$ Engle, ${ }^{16}$ and Peng and Qiu ${ }^{17}$ continue their studies on Ni-catalyzed difunctionalization of olefins by using fluorinated congeners, heteroleptic complexes or dihalogenated building blocks, accessing molecules that would be beyond reach otherwise. Fu and Wang describe the utilization of $\mathrm{N}$-acylsaccharins in a rather intriguing oxidative transamidation of tertiary aromatic amines, ${ }^{18}$ while Gong $^{19}$ and Newhouse ${ }^{20}$ report the utilization of Ni catalysts in broadly applicable hydrodeoxygenation of alkyl oxalates and benzylic dehydrogenation events.

Following up their ongoing interest in the field, Jarvo describes a new protocol for streamlining the preparation of fluorinated cyclopropanes, ${ }^{21}$ whereas Zhu and He describe the ability of ancillary ligands to dictate the site-selectivity in a Ni-catalyzed reductive hydroarylation of styrene motifs. ${ }^{22}$ Chu, $^{23}$ Fensterbank, $^{24}$ Martin $^{25}$ and Amgoune ${ }^{26}$ merge photoredox catalysis and Ni catalysts to enable dual functionalization of vinyl boranes, monoalkylation of dichlorostyrenes, photodehalogenation of organic halides, and $\mathrm{sp}^{3} \mathrm{C}-\mathrm{H}$ acylation events with $\mathrm{N}$-acyl imides. Last but not least, Diao offers a versatile tool for synthetic organic and organometallic chemists by tabulating experimentally measured redox potentials of Ni complexes supported by commonly employed ligands. ${ }^{27}$

As judged by the wealth of recent literature data on $\mathrm{Ni}$ catalysis, it is evident that this area of expertise has become an indispensable tool for the ever-expanding repertoire of our synthetic arsenal when forging $\mathrm{C}-\mathrm{C}$ and $\mathrm{C}$-heteroatom bonds. Undoubtedly, the meteoric development of metallaphotoredox catalysis and the ability to modulate the properties of Ni catalysts by suitable ligand modulation have of- fered conceptually new pathways for molecular assembly and 'top-down' strategies to explore currently inaccessible chemical space. Indeed, $\mathrm{Ni}$ catalysis has recently been adopted in late-stage functionalization as a strategy to boost lead generation approaches in the early phases of drug discovery programs. Therefore, it is inevitable to predict that $\mathrm{Ni}$ catalysis is in the midst of a transition that might well impact the practice of organic chemistry for years to come.

\section{References}

(1) (a) Corriu, R. J. P.; Masse, J. P. J. Chem. Soc., Chem. Commun. 1972, 144. (b) Tamao, K.; Sumitani, K.; Kumada, M. J. Am. Chem. Soc. 1972, 94, 4374.

(2) Ishida, N.; Kamino, Y.; Murakami, M. Synlett 2021, 32, DOI: 10.1055/a-1282-9731.

(3) Lundrigan, T.; Tassone, J. P.; Stradiotto, M. Synlett 2021, 32, DOI: 10.1055/a-1337-6459.

(4) Diao, H.; Shi, Z.; Liu, F. Synlett 2021, 32, 1494.

(5) Li, Y.; Yin, G. Synlett 2021, 32, in press.

(6) Wei, S.; Mao, Y.; Shi, S.-L. Synlett 2021, 32, DOI: 10.1055/a1379-1584.

(7) Minami, H.; Nogi, K.; Yorimitsu, H. Synlett 2021, 32, 1542.

(8) Kathe, P. M.; Berkefeld, A.; Fleischer, I. Synlett 2021, 32, DOI: 10.1055/s-0040-1706683.

(9) Shuai, B.; Fang, P.; Mei, T.-S. Synlett 2021, 32, DOI: 10.1055/s0039-1690872.

(10) Lin, Z.; Lan, Y.; Wang, C. Synlett 2021, 32, 1547.

(11) Ashida, K.; Hoshimoto, Y.; Ogoshi, S. Synlett 2021, 32, 1537.

(12) Luo, Y.; Chan, B.; Fukuda, T.; Onodera, G.; Kimura, M. Synlett 2021, 32, 1551.

(13) Chen, Q.; Fu, L.; You, J.; Nishihara, Y. Synlett 2021, 32, 1560.

(14) Iizumi, K.; Kurosawa, M. B.; Isshiki, R.; Muto, K.; Yamaguchi, J. Synlett 2021, 32, 1555.

(15) Zhao, Q.-W.; Yang, Z.-F.; Fu, X.-P.; Zhang, X. Synlett 2021, 32, 1565.

(16) Kim, N.; Tran, V. T.; Apolinar, O.; Wisniewski, S. R.; Eastgate, M. D.; Engle, K. M. Synlett 2021, 32, 1570.

(17) Xiao, J.; Wang, Y.-W.; Qiu, Z.-P.; Peng, Y. Synlett 2021, 32, DOI: 10.1055/s-0040-1707188.

(18) Liu, S.; Yang, L.; Tao, J.; Yu, W.; Wang, T.; Fu, J. Synlett 2021, 32, DOI: 10.1055/a-1517-5895.

(19) Ye, Y.; Ma, G.; Yao, K.; Gong, H. Synlett 2021, 32, DOI: 10.1055/a1328-0352.

(20) Zhang, P.; Cantrell, R. L.; Newhouse, T. R. Synlett 2021, 32, DOI: 10.1055/s-0040-1720446.

(21) Lucas, E. L.; McGinnis, T. M.; Castro, A. J.; Jarvo, E. R. Synlett 2021, 32, 1525.

(22) Xue, Y.; Chen, J.; Song, P.; He, Y.; Zhu, S. Synlett 2021, 32, DOI: 10.1055/a-1523-3228.

(23) Feng, X.; Guo, L.; Zhu, S.; Chu, L. Synlett 2021, 32, 1519.

(24) Abdellaoui, M.; Millanvois, A.; Levernier, E.; Ollivier, C.; Fensterbank, L. Synlett 2021, 32, 1513.

(25) Higginson, B.; Sanjosé-Orduna, J.; Gu, Y.; Martin, R. Synlett 2021, 32, DOI: 10.1055/a-1457-2399.

(26) Kerackian, T.; Reina, A.; Krachko, T.; Boddaert, H.; Bouyssi, D.; Monteiro, N.; Amgoune, A. Synlett 2021, 32, 1531.

(27) Lin, Q.; Dawson, G.; Diao, T. Synlett 2021, 32, DOI: 10.1055/s0040-1719829. 